\title{
Peculiarities of the influence of high- and low-energy proton and electron irradiations on the characteristics of silicon solar cells
}

\author{
V.V. Chernenko \\ V. Lashkaryov Institute of Semiconductor Physics, NAS of Ukraine \\ 45, prospect Nauky, 03028 Kyiv, Ukraine, \\ Phone+380 (44) 525-5043, fax+380 (44) 525-5788; e-mail: vvch@isp.kiev.ua
}

\begin{abstract}
Experimental data on degradation of photovoltaic and photoenergetic characteristics of silicon solar cells exposed by high-energy electrons and protons as well as low-energy protons have been obtained. The previously proposed theoretical model that can describe degradation of the solar cell characteristics under the influence of irradiation, including that creating spatially inhomogeneous defect distribution over the structure thickness, has been experimentally confirmed. It was ascertained that in the cases of $1 \mathrm{MeV}$ energy electron and $20 \mathrm{MeV}$ energy proton irradiations, when there is a relatively homogeneous defect distribution over the silicon solar cell thickness, its shortcircuit current degrades faster than the open-circuit voltage. On the contrary, in the case of low-energy $0.1 \mathrm{MeV}$ proton irradiation, when the distribution of defects is spatially inhomogeneous, the open-circuit voltage degrades faster than the short-circuit current.
\end{abstract}

Keywords: silicon solar cell, irradiation, degradation.

Manuscript received 14.04.10; accepted for publication 08.07.10; published online 30.09.10.

\section{Introduction}

The problem of radiation resistance of semiconductor devices attracts an increased interest due to considerable changes in properties of semiconductors under irradiation, on the one hand, and the need of wide application of semiconductor devices under conditions of radiation, such as space, nuclear power plants, etc., on the other hand. Therefore, there is a need to increase the radiation hardness of silicon solar cells (SC) used the space applications.

The main manifestation of radiation damage in $\mathrm{SCs}$ is increase in the concentration of recombinationactive defects leading to a reduced efficiency of photovoltaic energy conversion in them. Investigation of radiation effects in silicon SCs showed that their radiation resistance depends on the energy and kind of radiation particles [1]. The reason for the damage is that the high-energy particles create numerous structural defects in the crystal lattice of semiconductor: vacancies, interstitial atoms, defect clusters and different types of vacancy-impurity complexes. Under certain circumstances, these defects can act as surface and bulk recombination-active centers and thus modify the electrical characteristics of semiconductor [1-5].

The exposure with high-energy protons and electrons is known to lead to a homogeneous distribution of radiation defects over the SC thickness, while the irradiation with heavy particles, such as protons, having the path length smaller than the SC thickness, results in distribution of radiation defects that can be inhomogeneous in depth. In [6], the influence of irradiation on the basic photovoltaic characteristics of silicon SCs, namely: the short-circuit current and the open-circuit voltage for arbitrary relation between the damaged layer depth, the diffusion length of minority carriers before the exposure, and the structure thickness, was theoretically studied. A theoretical three-layer model of heterogeneous recombination in depth was proposed. This model takes into account an increase of the number of defects created by a proton at the end of path length and can describe the degradation changes of the characteristics of silicon SCs under irradiation, which causes spatially heterogeneous distribution of defects. Using the proposed model, peculiarities of the influence of irradiation on the photoelectric characteristics of silicon SCs for highly and weakly absorbed particles were theoretically ascertained [6]. In particular, it was shown that the exposure of SCs with low-energy protons with a path length of several micrometers results in faster degradation of open-circuit voltage as compared to that of short-circuit current and that the result is just the contrary in the case of exposure with electrons or high-energy protons. In the 
intermediate case, i.e. when irradiating the silicon SCs with protons possessing the path length within the range 10 to 100 micrometers, the relative behaviour of these characteristics can be different and is determined by the path length of radiation particles, the heterogeneity of defect distribution and radiation dose.

The purpose of this work was to make experimental verification of the proposed theoretical model and to study the peculiarities of the influence of electron and proton irradiations on the basic photoelectric and photoenergetic characteristics of silicon SCs with diffusion-field surface barriers.

\section{Samples and experimental technique}

The experimental samples were highly efficient (external efficiency $\eta=14-16 \%$ under AM0 spectral conditions) silicon diffusion-field type SCs [7, 8] with bases of both types of conductivity, made of KDB-10 and KBE-2 types of silicon with the area $2.33 \mathrm{~cm}^{2}$.

SC samples were irradiated with the high-energy electrons possessing the energy $E=1 \mathrm{MeV}$ as well as with the high-energy protons possessing the energy $E=$ $20 \mathrm{MeV}$, which creates a practically uniform distribution of radiation defects over the depth of $\mathrm{SC}$, and with the low-energy protons with the energy $E=0.1 \mathrm{MeV}$, creating an inhomogeneous distribution of radiation defects over the depth of SC due to the path length of $0.1 \mathrm{MeV}$ energy protons not exceeding few micrometers in silicon $[3,4]$. The irradiation was directed onto the SC front side oriented perpendicular to the particle flow. The exposure doses were collected step-by-step.

Investigations of the degradation characteristics of SCs were performed at the Test Center for Solar Cells and Photovoltaic Batteries of V. Lashkaryov ISP, NAS of Ukraine, certified by the bodies of the State Consumer Standard of Ukraine [9]. The current-voltage characteristics under illumination were measured before irradiation and after each stage of exposure. From the measured current-voltage characteristics under illumination, the main photoelectric and photoenergetic characteristics of SCs, namely: the short-circuit current, the open-circuit voltage and maximum output power were calculated.

\section{Experimental results and discussion}

Typical dependences of the short-circuit current $I_{S C}$, open-circuit voltage $V_{O C}$ and maximum output power $P_{M}$ of diffusion-field type SCs with bases of both types of conductivity on the dose of electron flux with the energy $E=1 \pm 0.1 \mathrm{MeV}$ are shown in Fig. 1. It is known that the SCs with the $p$-type base have higher radiation resistance as compared to those with the $n$-type base [10]. Indeed, the results of irradiation experiments with electron fluxes showed that the $n$-base SCs made of KBE-2 silicon have lower radiation resistance as compared to that of $p$-base SCs. Already at the dose of $1 \mathrm{MeV}$ irradiating electrons $F_{e}=1 \cdot 10^{13} \mathrm{~cm}^{-2}$, the value of the current $I_{S C}$ of $n$-type base SC was decreased by $32 \ldots 34 \%$ and the value of voltage $V_{O C}$ - by $12 \ldots 13 \%$ with respect to their values before irradiation, respectively. These changes can be compared with the decrease of the values of these characteristics by 1 to $2 \%$ for $p$-type base SCs. Upon further increase of the dose, stronger dependences of the degradation degree for the current $I_{S C}$ and voltage $V_{O C}$ for the $n$-type base SCs (Fig. 1, curves 1 and 2) were observed, as compared to the $p$-base SCs (Fig. 1, curves 4 and 5). Due to this behaviour of these two characteristics at the dose $F_{e}=2 \cdot 10^{14} \mathrm{~cm}^{-2}$, the value of degradation of the maximum output power $P_{M}$ for $n$-base SCs was more than $60 \%$ against approximately $20 \%$ for $p$-base SCs (Fig. 1, curves 3 and 6). The results presented in Fig. 1 show that the SC current $I_{S C}$ degrades more rapidly than its voltage $V_{O C}$ with increasing the dose of high-energy electron irradiation.

Degradation changes of these SC characteristics under study had similar character in the cases of irradiation of SCs with bases of both types of conductivity with high-energy protons possessing the energy

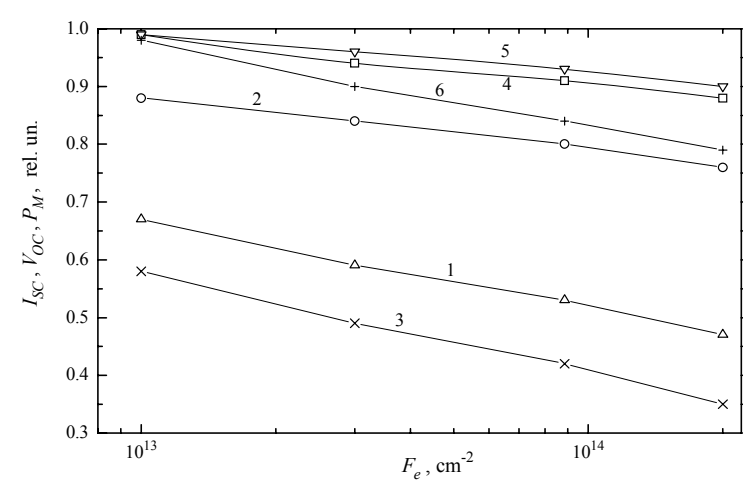

Fig. 1. Typical dependences of the short-circuit current $I_{S C}$ $(1,4)$, open-circuit voltage $V_{O C}(2,5)$ and maximum output power $P_{M}(3,6)$ normalized to the initial (before irradiation) values of respective variables for the SCs with the $n$ - $(1,2,3)$ and $p$-base $(4,5,6)$ versus dose of $1 \mathrm{MeV}$ electron fluxes.

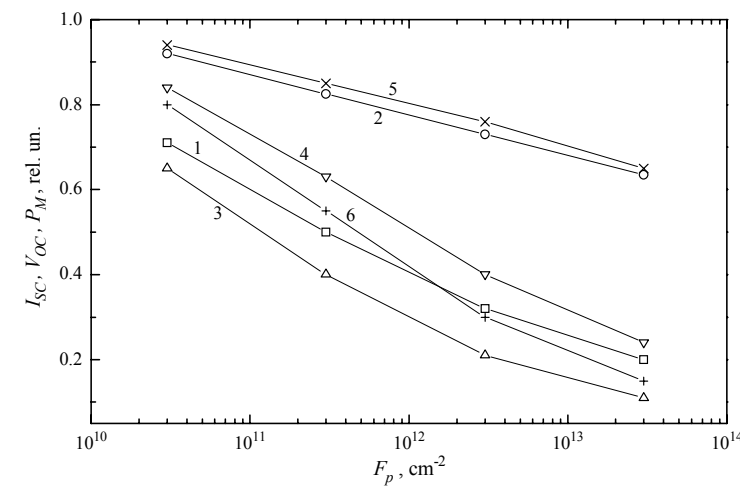

Fig. 2. Typical dependences of the short-circuit current $I_{S C}$ $(1,4)$, open-circuit voltage $V_{O C}(2,5)$ and maximum output power $P_{M}(3,6)$ normalized to the initial (before irradiation) values of respective variables for the SCs with the $n$ - $(1,2,3)$ and $p$-base $(4,5,6)$ versus dose of $20 \mathrm{MeV}$ proton fluxes. 


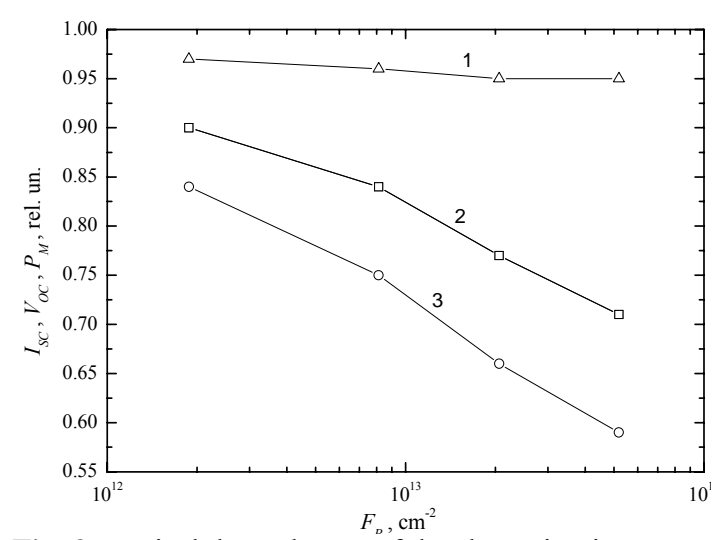

Fig. 3. Typical dependences of the short-circuit current $I_{S C}(1)$, open-circuit voltage $V_{O C}(2)$ and maximum output power $P_{M}$ (3) normalized to the initial (before irradiation) values of respective variables for the $p$-base $\mathrm{SC}$ versus the dose of $0.1 \mathrm{MeV}$ proton fluxes.

$E=20 \pm 1 \mathrm{MeV}$ (see Fig. 2). The main difference between the results of exposures with the high-energy electrons and high-energy protons was that the dose of protons required for the same degradation of the open-circuit voltage and short-circuit current of SC was around 3 to 4 orders of magnitude lower than that for electron irradiation. This is consistent with the results of other studies, according to which irradiation with the highenergy protons and electrons causes appearance of similar defects, the main difference being the different rates of such defect formation $[1,4]$. The SC current $I_{S C}$ degrades also more rapidly than its voltage $V_{O C}$ with increasing the dose in the case of high-energy $20 \mathrm{MeV}$ proton irradiation, similar to the case of $1 \mathrm{MeV}$ electron irradiation.

Typical dependences of normalized values for the open-circuit voltage and short-circuit current of SCs versus the dose of irradiation with the low-energy protons possessing the energy $E=100 \pm 0.1 \mathrm{keV}$ are presented in Fig. 3. These results show that unlike the case of high-energy proton irradiation, the open-circuit voltage decreases more rapidly than the short-circuit current with increasing the dose of low-energy proton irradiation when the defect layer created by radiation is located within a relatively narrow frontal near-surface region of $\mathrm{SC}$.

\section{Conclusion}

The peculiarities of the influence of irradiations with the high-energy and low-energy protons and electrons on characteristics of silicon SCs were experimentally studied. It was ascertained that in the case of high-energy radiation, when the radiation particles pass through the entire SC and create a relatively homogeneous distribution of defects over its thickness, there takes place more rapid degradation of the short-circuit current in SC as compared to its open-circuit voltage. In the case of exposure with low-energy protons, when the radiation particles create a spatially inhomogeneous defect distribution with defect localization within the frontal near-surface region of SC, there takes place more rapid degradation of the opencircuit voltage of SC as compared to its short-circuit current. The experimental data on radiation induced degradation of the photovoltaic characteristics of SCs obtained in this paper are qualitatively consistent with the results of the theoretical analysis performed earlier [6].

\section{References}

1. A.M. Vasil'ev, A.P. Landsman, Semiconductor Photoconverters. Soviet Radio, Moscow, 1971 (in Russian).

2. I.D. Konozenko, A.K. Semenyuk, V.I. Khivrich, Radiation Effects in Silicon. Naukova Dumka, Kiev, 1974 (in Russian).

3. N.V. Kuznetsov, G.G. Solov'ev, Radiation Hardness of Silicon. Energoatomizdat, Moscow, 1989 (in Russian).

4. H.Y. Tada, J.R. Carter, B.E. Anspaugh, R.G. Downing, Solar Cell Radiation (Handbook). Jet Propulsion Laboratory Publication, Pasadena, California, 1982.

5. K. Nishimura, M. Yamaguchi, O. Anzava et al., Effect of impurities on generation of radiationinduced defects in Si single crystals and space solar cells // Proc. 3-rd World Conf. on Photovoltaic Energy Conversion, Osaka, Japan, 2003, p. 3O-D6-03.

6. A.P. Gorban, N.A. Prima, A.V. Sachenko, V.P. Kostylyov, V.V. Chernenko, Effect of radiation on silicon solar cells. Theoretical modelling effects associated with spatial distribution of radiation defects // Optoelektronika $i$ poluprovodnikovaya tehnika, 36, p. 57-64 (2001) (in Ukrainian).

7. A.P. Gorban, V.P. Kostylyov, A.V. Sachenko, A.A. Serba, V.V. Chernenko, Development physical and technical bases for creating highefficiency silicon solar photoconverters and batteries for cosmic and terrestrial applications // Collection of Scientific Papers: Aerospace engineering and technology, 8, p. 83-87 (1999), in Russian.

8. A.P. Gorban, V.P. Kostylyov, V.G. Litovchenko, A.A. Serba, V.V. Chernenko, High-efficiency diffusion-field silicon solar cells with thermally oxidized surface // Proc. 6 Republican Conf. "Physical problems of MIS integrated electronics", Sevastopol', Ukraine, 1990, p. 58 (in Russian).

9. Attestation Certificate of Test Center for Solar Cells and Photovoltaic Batteries of V. Lashkaryov Institute of Semiconductor Physics, NAS of Ukraine, №PT-102/10. Issued April 1, 2010 by State Enterprise Ukrainian State Research and Production Center of Standardization, Metrology, Certification and Consumer Protection.

10. A. Fahrenbruch, R. Bube, Solar Cells. Theory and Experiment. Energoatomizdat, Moscow, 1987 (in Russian). 